\title{
El origen de la propiedad y su legitimación: Un recuento histórico
}

\author{
The Origin of the Property and its Legitimation: A Historical Overview
}

Niklas Luhmann

Facultad de Sociología, Universidad de Bielefeld, Alemania

\section{RESUMEN}

En este artículo Niklas Luhmann propone describir la propiedad considerando la forma de operar de la sociedad funcionalmente diferenciada. Descripción que se confronta con aquellas semánticas que explicaron el problema de la propiedad como un fenómeno político, jurídico, o producto de la ideología burguesa. Para Luhmann, la propiedad es el aspecto constitutivo del sistema de la economía, que ha de entenderse como un sistema funcionalmente diferenciado, clausurado autopoiéticamente, orientado por su propio código y programas. Precisamente, tal autonomía posibilita que la propiedad llegue a concebirse también como un concepto jurídico o que constituya una motivación para la toma de decisiones políticas. Desde esta perspectiva, el problema de la propiedad no puede continuar fungiendo como fundamento explicativo de la sociedad, sino que por el contrario, la propiedad ha de entenderse en el marco de una teoría general de los sistemas sociales.

PALABRAS ClaVE: Sistema social; Semántica; Propiedad; Economía; Derecho

\section{ABSTRACT}

In this paper Niklas Luhmann propounds to describe the property in consideration of the operation form of the functional differentiation society. Description than debates with those semantics that explained the property problem as a political or legal problem, or as a bourgeoisie ideology product. According to Luhmann, the property is the basal attribute of the economy system, that is a functional differentiation system, oriented by its own code and programs. Precisely, this autonomy make possible the property as a legal concept, or as a politic decisions motivation. In this approach, the property problem can no longer be a explanatory basis of the society, but, the property must be understood within the scope of a Social Systems' General Theory.

KEYWORDS: Social system; Semantics; Property; Economy; Law

$I^{*}$

Debemos partir del hecho de que la sociedad moderna no ha encontrado todavía una teoría adecuada de sí misma. Descripciones como sociedad capitalista, industrial o postindustrial, civilización tecno-científica, post-

\footnotetext{
* Traducción de Raúl Zamorano Farías, Giancarlo Corsi y Rogelio Salgado. Título original: Der Ursprung des Eigentums und seine Legitimation. In: W. Krawietz et al (Hrsg.), Technischer Imperativ und Legitimationskrise des Rechts, Rechtstheorie Beiheft 11, Berlin, 1991. Resúmenes y palabras claves añadidos por los traductores.
}

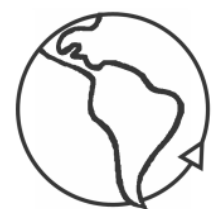

REVISTA MAD - UNIVERSIDAD DE CHILE

Revista del Magíster en Análisis Sistémico Aplicado a la Sociedad

Facultad de Ciencias Sociales, Departamento de Antropología

Universidad de Chile

ISSN 0718-0527

www.revistamad.uchile.cl 
moderna, etcétera, se multiplican. Esto implica que la comprensión de muchas de nuestras interpretaciones históricas se impongan a conveniencia mas que no puedan ser verificadas, porque a tal fin sería necesaria una teoría que no tenemos.

En esta situación, los trabajos hacia una teoría de la sociedad moderna tienen prioridad sobre cualquier otra empresa sociológica. Pero también es útil -y esta es una tarea más delimitada- tomar en consideración el contexto histórico que ha dado y da todavía forma a la comprensión de muchas instituciones. La cuestión de la propiedad, y especialmente la cuestión de la propiedad privada, es particularmente importante sobre todo porque en referencia a ella en el siglo XIX se han articulado ideologías todavía citadas a finales del XX. Los varios sistemas que tratan la propiedad llevan a diferencias y a tensiones de relevancia mundial, a la desconfianza y al peligro de guerras, como es el posible seguir temiendo. Por tanto no es cosa de poca importancia buscar entender que no solo una guerra sería insensata, sino también sus motivos.

Mi tesis de partida es que en el siglo XVII inician a mostrarse los primeros tratados de una percepción de las relaciones modernas y que en la segunda mitad del XVIII se impone la idea que la Europa moderna se distingue de cualquier otra sociedad en la comparación sea histórica, sea regional. La semántica, con la cual es elaborada esta idea, y las palabras clave abarcan desde la idea de Estado constitucional hasta el amor romántico, desde la pedagogía de la formación a la positividad del derecho, desde la física newtoniana a la libre economía de mercado; esta semántica, sin embargo, puede tener solo un significado transitorio. Ella describe la sociedad moderna en una fase de transición hacia un futuro abierto, indeterminado, que se perfila como positivo. Dado que el hombre así se considera, es constructor de su mismo futuro, las cosas solo pueden ir mejor, si se opera en modo correcto. Si se consideran las consecuencias de las estructuras de la sociedad moderna, por ejemplo aquellas de la industrialización, la mirada se hace repentinamente profunda; pero al inicio solo sobre la base de problemas relativamente concretos y cercanos a la cotidianidad.

Es hora de sustituir esta semántica transitoria, desearía decir esta semántica del Medioevo moderno. Pero antes de proceder debemos conocerla mejor. No vivimos en absoluto en una época postmoderna. La fuerza arrastrante de las condiciones estructurales que caracterizan la sociedad moderna continúa operando sin interrupción y de modo casi ciego, sea lo que sea que piensen los intelectuales. Pero ella ha conducido al sistema de la sociedad a una situación muy distinta de aquello que se esperaba como 
progreso. Por esto, la distancia que hemos alcanzado al final de este siglo nos permite observar retrospectivamente aquella semántica transitoria dando un vistazo sea al tardío derecho natural y al iluminismo de la razón, sea al choque ideológico del siglo XIX, que se había alcanzado entre otras cosas a través del problema de la propiedad.

En el pasaje de una sociedad diferenciada primariamente en sentido estratificado según las clases y secundariamente según centro/periferia (ciudad/campo) a una sociedad funcionalmente diferenciada, no se podía ver hacia donde se estaba yendo. El presente no ofrecía suficientes puntos de referencia para reconocer el futuro. Era necesario por el contrario atenerse a condiciones por así decir manifiestas. Así se explica por ejemplo la sorprendente confianza en la moral que en torno al siglo XVIII regresa a Europa después de un siglo y medio de escepticismo y de tentativas para evitar conflictos. Algo similar se puede decir para el significado paradigmático de la matemática y de las leyes naturales, para la interpretación de la naturaleza del hombre como razón. A esto nos podíamos atener de frente a los cambios de la estructura de la sociedad o al menos se podía creer y esperar que esto bastase. Mi tesis es que precisamente este contexto ha dado a la cuestión de la propiedad su significado para la definición y la justificación de la sociedad civilizada, como se dice ahora.

II

Si se observan las teorías de la propiedad desarrolladas en el siglo XVII y en el temprano XVIII, es fácil ver que la máxima forma de la vida humana, la sociedad política o la sociedad civil, es entendida como una sociedad de propietarios. Es entonces una sociedad que no incluye a cualquiera (si no indirectamente mediante la dependencia doméstica): no se es miembro de esta sociedad simplemente en cuanto seres humanos, sino solo bajo condiciones particulares, de las cuales derivan derechos y deberes. Esto vale en ambos casos, sea que se piense la sociedad como fundada sobre el contrato, sea que se utilice la metáfora del organismo. Tampoco conceptos como populus, people, peuple, en su uso lingüístico referido a la sociedad, indican a cualquiera, sino solo a aquellos que portan en la sociedad sus intereses particulares y en este modo participan de los derechos y de los deberes. Para quien no es autónomo el sistema social competente es la casa, no la sociedad civil, y aquí se trata de economía, no de política.

Empero esta semántica tradicional veteroeuropea, no se puede dejar de ver que el poder político en el sistema que ahora es indicado como Es- 
tado, por cuanto aun en un sentido vago, no es simplemente una derivación de la propiedad. La propiedad de la casa del señor es diferenciada en sentido sea contable, sea jurídico, del poder del señorío. En un modo igualmente claro, en la praxis de los "estados soberanos", en el curso del siglo XVII se impone también el hecho de que la propiedad de la tierra en cuanto tal no implica todavía poder sobre quien la habita, pero cada sentencia y cada cuestión administrativa -también esto es ahora por primera vez claramente diferenciado- debe ser reconducida al estado y practicada como poder delegado según disposiciones que el poder central puede emanar y cambiar. Una vieja distinción, aquella entre dominium e imperium, adquiere así un peso nuevo y sirve ahora para encauzar ulteriores diferenciaciones estructurales entre economía y política.

Un debate entre teoría política y teoría de la propiedad muestra muy pronto que ya en el siglo XV, si no antes, ambas representaciones de la sociedad emprenden caminos diversos. La cuestión de la fundación es puesta como cuestión del origen, y una vez puesta de ese modo, no puede dársele una respuesta unitaria. En el curso del siglo XV, en la época de la contraposición con las teorías del Movimiento Conciliar y de las doctrinas tardo medievales de la soberanía popular, para el poder político (imperium, potestas) es desarrollada una aproximación jerárquica. Orden y jerarquía. Pero la jerarquía es algo más que una mera subdivisión en rango y grado y también, al menos en el entendimiento europeo, algo más que una mera diferenciación de la sociedad. La jerarquía funda el poder político, sea en la iglesia, sea en los estados territoriales que se están desarrollando. El principio de la jerarquía se funde entonces con el principio monárquico. El orden es por tanto fundado desde lo alto, es por así decir constituido a partir del vértice y los defensores extremos de esta teoría declaran también que la jerarquía fundaría la sociedad y no la sociedad la jerarquía.

Aunque teorías del género son desarrolladas para fundar y reforzar la posición del papa en la iglesia, terminan por ser rápidamente generalizadas al poder monárquico en cuanto tal y transferidas sobre el estado territorial. Los sistemas políticos europeos, también en las repúblicas citadinas italianas, tienden a la monarquía. No podemos profundizar aquí las teorías relativas a estos desarrollos; para nuestro tema es decisivo solo el hecho de que ellas constriñen a separar política y economía. La aproximación jerárquica puede ser ciertamente trasladada desde la iglesia al estado, pero no es adecuada para representar las relaciones de propiedad. Si la teoría política hace derivar del vértice el imperium o la potestas del poder político (y entonces quita vigor a las teorías basadas sobre el contrato y 
sobre la delegación), ella lleva inevitablemente a un cisma semántico porque la posesión y la disposición sobre la propiedad no pueden ser fundadas en este modo. En la teoría de la propiedad es necesario entonces buscar otras fundaciones, según otro modo de "origen" de la propiedad, y la respuesta se encuentra en la doctrina de una propiedad común originaria, pero luego disuelta por motivos racionales.

Relativamente separada de la teoría política de la propiedad, también la doctrina jurídica de la propiedad introduce innovaciones, la más importante de las cuales es tal vez aquella que, a partir de Bartolo, pone en evidencia el momento de la facultad de disponer de ella. En este modo la doctrina de la propiedad puede ser adaptada a la economía monetaria, pero por otra parte esto conlleva problemas de justificación, porque si en el pensamiento antiguo el gusto por la propiedad (fruitio) parecía bastar para legitimar la propiedad, el derecho a la disposición inserta en la institución un momento de negativa, un momento de incerteza, de riesgo. ¿Cómo se puede justificar la propiedad con base en el hecho de que se puede disponer a placer o aún más que se la pueda ceder a placer?

No obstante la centralidad de esta pregunta, la literatura del periodo aquí considerado, entre los siglos XVII y XVIII, no ve en esto el verdadero problema. Ella lo remueve sustituyéndolo con otro y precisamente con la cuestión del origen y de la subdivisión de la propiedad. La necesidad de la disponibilidad económica es tan obvia que es disuelta dentro del concepto de propiedad. Instalando la cuestión de la fundación de la propiedad se formulan otros problemas.

Para los juristas es importante antes que nada solo la prueba de que la propiedad haya sido heredada legalmente. Esto puede tener lugar solo en modo que no perjudique derechos de otros. Normalmente se hereda legalmente la propiedad con el traspaso de otro propietario. Pero así la cuestión de la herencia legal solamente se desplaza. Para aclarar el origen de la propiedad no es suficiente. Por esto basándose sobre fuentes bíblicas y de otra proveniencia, se parte de una propiedad originaria común que en un cierto momento ha sido dividida mediante un contrato o tácitamente. Se completa también pensando en la posibilidad de acceder a bienes carentes de dominio como forma de apropiación primaria. Como sea, la legitimación de la propiedad no es derivada de las modalidades de utilización de la sociedad del tiempo, sino es reconducida a la transformación de un estado originario. Esta transformación es pensada como transformación legal, y de esto depende el argumento. El originario estado de naturaleza era legal, su transformación era legal, así como lo es la diferencia 
entre razón y daño o entre legal e ilegal que deriva y que se articula basándose sobre la propiedad individual. Puedo excluir a otros de mi propiedad de manera legal y si ellos no están de acuerdo entonces están en la ilegalidad.

¡Curioso! La diferencia entre legal e ilegal es a su vez legal. Se pueden legalmente empujar otros a la ilegalidad. Desde cualquier punto de vista debe haber sido un embrollo lógico pero la problemática relativamente concreta de la propiedad y del retorno a su necesidad y a su utilidad social esconden el problema.

El esfuerzo hecho delata un cierto malestar con las soluciones. Un contrato tácito bastaría para disolver la propiedad común -como si una mera participación en la vida social significase resignarse a la división de la propiedad, con el mismo significado de un acuerdo contractual. Pufendorf, refiriéndose a Grozio, propone considerar la originaria propiedad común como una communitas solo negativa, en la cual ninguno podría excluir a otros de algo, pero ninguno podría heredar derechos contra otros entonces como un estado societario sin diferencia entre legal e ilegal. Esto, en el siglo XVIII, deviene doctrina dominante y documenta aquellos que se quiere mostrar: que es posible heredar propiedad sin ilícito. Para el derecho natural alemán-latino es además indicativo el hecho de que el derecho es construido a partir del deber (officium, obligatio). Esto permite una combinación con el utilitarismo del siglo XVIII. La propiedad conlleva el deber (si bien solo como obligación natural) de actuar en modo útil en la sociedad. El viejo doble significado del latín abuti como consumo y abuso es disuelto. Contra el consumo racional de propiedad no se puede objetar nada, naturalmente, pero se puede contra el abuso, contra el derroche inútil o también contra la no utilización para aumentar el bienestar.

Me detengo aquí con estos retornos a los problemas conceptuales de la construcción jurídica. Lo que es interesante sociológicamente es el hecho de que aquí, con todo el refinamiento propio de la competencia profesional, se elabora una respuesta a un problema estructural de la sociedad. Si Marx hubiese vivido cien años antes, habría escrito una crítica del derecho natural político.

Y en lugar de ello se lee en Rousseau, en el famoso inicio de la segunda parte del discurso sobre la desigualdad: Le premier qui avant encols un terrain, s'avisa de dire, ceci est à moi, et trouva des gens assés simple pour le croire fut le vrai fondateur de la société civile. El fundamento de la propiedad es la estupidez de aquellos que la aceptan. Y un poco más tarde Linguet y 
otros dirán: el fundamento de la propiedad es la violencia de la apropiación.

Ahora, esto no es mejor que la doctrina imperante, es solo darle la vuelta. La teoría burguesa se presenta muy pronto de forma doble: Christian Wolff y Rousseau, Pothier y Linguet, Ricardo y Marx. Pero hoy debemos preguntarnos, y con esto regreso al inicio de mi exposición, si esta aproximación de la teoría burguesa sea suficiente. ¿Se debe, se puede decidir al interior de esta aproximación para la variante positiva o para la negativa? Pero si la sociedad es la unidad de ambas, ¿no es en cambio pertinente repensar en nuevo modo toda la problemática?

\section{III}

Las teorías de la propiedad vistas hasta ahora se leen como si se tratase de propiedad de la tierra o de bienes inmuebles. También los ejemplos que se encuentran en la literatura de la época muestran que se entendía esto. ¿Se puede cercar un terreno que no pertenece a ninguno (o a todos) y declararlo propiedad privada? ¿Se puede considerar un pavorreal, que pasea en un campo, como bien sin dominio y apropiárselo? ¿Pero es posible que los juristas no se hayan percatado de que vivimos desde hace tiempo en una economía determinada por el dinero, que incluso la propiedad de la tierra se puede comprar y que por dinero se puede trabajar o hacer trabajar a cambio de una compensación? Ciertamente, por esto el derecho regula el comercio y el arrendamiento, el alquiler y las relaciones de trabajo dependiente; pero que el dinero amenace el sentido de la propiedad, la vuelva mercancía, determine su valor, no parece afectar al concepto y las teorías de la propiedad. El dinero es tratado como una cosa entre otras, para la cual valen las reglas de la propiedad de bienes inmuebles. Si la diferenciación societaria es concebida y justificada como diferenciación de la propiedad, el dinero es una cosa entre otras. Es interesante que el concepto de riqueza se encuentra casi siempre al plural: riquezas, richesse, riches, y claramente se piensa en bellos vestidos y palacios, buena comida y buenas ganancias. Y solo esta referencia parece dar a la cuestión de la propiedad aquella evidencia, aquella manejabilidad, aquella tangibilidad que ofrece un asidero suficiente en una sociedad que cambia rápidamente, no por último por vía de los efectos de la economía monetaria, y evoluciona hacia formas todavía desconocidas.

Existe, sin embargo, una excepción importante, cuyos efectos se prolongan todavía por cerca de un siglo y en efecto se expresaran plena- 
mente solo en las teorías socialistas de las primeras décadas del siglo XIX. Pienso en la teoría de la propiedad de los Two Treatises of Government de John Locke (1690).

También aquí la justificación de la propiedad no se basa sobre el dinero, sino sobre la producción de bienes mediante el trabajo. La inocencia con la cual todo comienza, se coloca aquí en el derecho indiscutible a la actividad corporal, al aumento del propio bienestar, a la producción de cosas útiles. Casi todo valor surge del trabajo y ¿quién quisiese dudar que el resultado del trabajo corresponde a quién lo ha generado? Si se lo quiere arrebatar, se da lugar a un ilícito. También aquí, entonces, el derecho natural y libre de ilegalidad produce la diferencia entre legal e ilegal. También aquí un relato casi mitológico. También aquí la referencia a la teología es precisamente a través del arquetipo del Dios "workmanship". La propiedad común permanece presupuesta como el ámbito del cual el trabajo puede extraer su propio material. Si no basta, se lo puede comprar. El acento, sin embargo, no es puesto sobre esto, sino sobre la creación de valor mediante trabajo. No se trata simplemente de la división de una cantidad de bienes ya constantemente dada, sino de formas de participación en el aumento del bienestar.

Todo esto puede ser formulado antes que nada sin referirse al dinero. La idea de un aumento en línea de principio ilimitado del bienestar puede darse sin embargo solo en una economía de mercado. Y entonces se pueden hacer trabajar a otros pagándoles y absorber la utilidad excedente del salario. Es luego cuestión de cálculo racional si es mejor producir todo solo o si sea mejor someterse a las condiciones de la división del trabajo si se compra la utilidad del trabajo de otros. Llegamos a Adam Smith y David Ricardo. En lugar de la inocencia del procedimiento que genera diferencias queda la racionalidad en el tratar la diferenciación societaria. El mismo Locke, si se puede decir así, ve que llega la infelicidad: "Find out something that hath the use and value of money amongst his neighbours, you shall see the same man will begin presently to enlarge his possessions", se lee en el Treatise (Libro II, par. 49). Pero dado que esto funciona con el dinero, está justificado por el consenso. El dinero puede circular solo sobre la base del consenso y la circulación queda en lugar del reclamo a un pactum explícito o tácito. Se notan enormes diferencias entre ricos y pobres y se las registra. La teoría las registra y tal vez las estigmatiza. Pero no persigue la intención de trasladar a la ilegalidad aquello que más tarde

\footnotetext{
*En inglés en el original (NdT).
} 
se habría llamado "commercial society" y luego sociedad capitalista. Ella se limita a adecuar la teoría del origen sin ilegalidad en un modo que pueda resultar evidente también dadas las condiciones de la economía monetaria.

\section{IV}

Retrospectivamente, en esta doctrina de la propiedad sorprende sobre todo el hecho de que se trabaja con la distinción entre estado de naturaleza y estado civilizado, esto es con una distinción entre pasado y presente. Falta un concepto para el futuro. El futuro no permanece indeterminado pero es insertado en el presente mediante esperanzas de progreso y sobre todo, desde el siglo XVIII, mediante la expectativa de un bienestar creciente. Su punto de anclaje en el presente está en la idea de utilidad. Las cuestiones jurídicas terminan siempre más bajo la prospectiva de cuál solución prometa la mayor utilidad posible y la misma moral "eudemonística" permite toda acción que no perjudique a ninguno. Aquello que está permitido y aquello que está prohibido no derivan de una descripción de la sociedad. Más bien la sociedad es vista como resultado de la investigación de la utilidad. La idea de un orden estratificado jerárquico sobrevive aún y en cuanto teoría de la sociedad no es sustituido todavía. Es impulsada por la dinámica de la investigación de las utilidades, en parte por la praxis decretista de los Estados, en parte por la liberalización de la maximización individual de las utilidades. Se ve claramente: en una sociedad aún tardomedieval se está preparando una nueva.

En muchos aspectos la distinción entre estado de naturaleza y estado civilizado toma el lugar de una teoría de la sociedad. Esto es evidente en el hecho de que ella no es entendida como una descripción solo histórica. A menudo la confrontación entre estado de naturaleza y estado civilizado justifica la sociedad moderna y esto de nuevo por cuanto refiere a las utilidades. El estado civilizado permite una explotación mejor de los recursos y permite una "vida mejor" (concebida no ya en el sentido aristotélico, sino neoeconómico). En el curso del siglo XVIII, sobre todo a partir de Hume, avanza ciertamente una historización que busca rastrear el estado de naturaleza en los salvajes entonces vivientes o en las fuentes históricas. No apenas sucede esto, la distinción pierde su función de justificación. Deviene nostálgica y la descripción del estado civilizado se vuelve así ambivalente. Se considera indispensable aquello que se ha obtenido y al mismo tiempo se reclama aquello que se ha perdido. También en este 
sentido la teoría burguesa muestra ya, en sus inicios, una doble valoración de la modernidad -pero tal vez solo porque la sociedad moderna no puede ser descrita en modo adecuado y entonces la descripción es sustituida por una valoración completa, es decir, positiva y negativa.

Un segundo cambio se dirige más allá de la discusión jurídica del concepto de propiedad, pero tiene efectos sobre ella. La propiedad había sido una justificación de la sociedad política, dado que ésta era constituida por economías domésticas. Desde el siglo XVII se abre camino el concepto de economía política (que en la antigüedad no era utilizado). Al mismo tiempo, siempre al inicio del siglo XVII, se desarrolla una teoría económica que va más allá de las doctrinas relativas a la economía doméstica, la cual investiga dependencias nacionales e internacionales. Desde la segunda mitad del siglo XVIII esta nueva teoría económica asume la función de teoría de la sociedad, como si la sociedad humana fuese primariamente, si no exclusivamente, una empresa económica. La sociedad, según esta concepción, regula la satisfacción de las necesidades humanas y a tal fin introduce la división del trabajo y la propiedad. En este modo la discusión jurídica sobre el concepto de propiedad es imprevistamente descargada del peso de justificar la sociedad de los propietarios. En Adam Smith este lugar lo toma la teoría de la commercial society que se distingue del estado de naturaleza por la división del trabajo. Las formas jurídicas de la propiedad pueden solamente ser dejadas a la comparación estoica y se vuelve evidente que la economía basada sobre la división del trabajo no es la ejecución de una ley de naturaleza (con sanción mediante ilícito), sino que las instituciones de la propiedad deben ser adaptadas a las exigencias de la división del trabajo (con sanción mediante insuficiente explotación de las posibilidades económicas).

De este modo, el derecho natural es relevado de la tarea de ofrecer una teoría normativa de la sociedad. La propiedad puede ser entonces concebida como institución juspositivista. Libertad y limitación de las facultades dispositivas de los propietarios devienen el instrumental técnicojurídico con el cual el derecho se adecua a las exigencias de la sociedad; y "exigencias de la sociedad" significa ahora: exigencias económicas. A un Hegel esto puede no bastar. A un Marx esto basta.

\section{$\mathrm{V}$}

Hasta aquí el concepto de propiedad ha permanecido como un concepto central de la teoría de la sociedad -sea políticamente con referencia a la 
propiedad de la tierra; sea jusnaturalísticamente y jurídicamente referencia a la exclusividad de las facultades dispositivas; sea, en fin, económicamente con referencia a la mejor institución posible de crecimiento económico. Proporcionalmente a la realidad de la sociedad moderna, estas teorías han resultado poco convincentes. Hoy, retrospectivamente, se pueden comprender como semánticas de transición, pero no nos dicen nada sobre la realidad de la sociedad moderna.

La afirmación de la teoría burguesa del siglo XVIII, según la cual la Europa moderna tendría más ventajas y más desventajas que otras sociedades, es tan poco manejable cuanto una pieza de pan con mantequilla por ambos lados. En comparación, la disolución de este problema en controversias ideológicas es ciertamente un progreso: se puede estar de acuerdo sobre la necesidad de cambiar la sociedad actual, pero discrepar sobre el camino hacia el futuro. Para unos, los sucesos obtenidos hasta ahora de la sociedad de los propietarios privados permiten tener esperanzas. Su receta es: avanzar con siempre nuevas posibilidades científicas o técnicas. Para los otros, existe necesidad de una revolución para llevar esta sociedad a la propiedad común, regresando entonces a la ambivalencia de una communitas sea positiva, sea negativa, en todo caso natural y sin derecho.

Las posibilidades de construir una teoría de la sociedad a partir de la propiedad parecen así estar al máximo. En todo caso, sobre este plano no han surgido ideas nuevas desde hace más de cien años. Ninguna duda, naturalmente, de que el modo en el cual se regula la utilización de la propiedad tiene un significado considerable y precisamente tanto para el sistema de la economía cuanto para el político. Si la propiedad de los medios de producción es estatalizada, el sistema político es responsabilizado en medida sin precedentes (cargándolo con dimensiones del todo nuevas de responsabilidad política) y hasta ahora no existen ejemplos de que esta responsabilidad en la forma de un sistema pluripartidista se pueda combinar con la posibilidad de un cambio de gobierno mediante elecciones políticas: también las tentativas de los países del este de reformar la economía mostrando que la economía mantiene la propia dinámica igualmente en estas condiciones y que no puede ser concebida como resultado maleable de las decisiones políticas. No apenas es sustituida una economía monetaria y hasta que ella existe, la economía depende de motivos para ganar y gastar dinero y estos motivos no se pueden crear o sustituir con el derecho o con apelaciones políticas. Una economía monetaria puede dar motivos al propio movimiento solo por sí misma y la cuestión es solamente: si y 
cuánto la propiedad de medios materiales o monetarios sea indispensable a tal fin.

En el contexto de esta relación no se trata, sin embargo, de cuestiones de teoría económica. Mi tesis es que ni la teoría jurídica de la propiedad, ni la económica ofrecen un concepto suficiente de sociedad moderna. La propiedad tiene la ventaja de la evidencia, ventaja que aconsejó, en el pasaje a formaciones societarias todavía desconocidas, confiarse a ella. La propiedad y sus efectos consiguientes como riqueza y pobreza o también trabajo y desocupación, se pueden observar bien. Referirse a ellos es indiscutiblemente evidente. También su condicionamiento y su relevancia son indiscutibles. Pero como teoría de la sociedad esta focalización no basta, porque existen indiscutiblemente muchas otras circunstancias societarias que a partir de ahí o se pueden entender o de cualquier forma no de modo suficiente.

La unilateralidad de una descripción de la sociedad que tiene de fondo la cuestión de la propiedad no puede ser superada confiándose en otras características de este género. En cambio, no basta hablar de propiedad, de civilización técnico-científica o bien, como hace hoy día Anthony Giddens, de política ordenada por Estados territoriales. Se podría referir también a la educación extrafamiliar desplazada a las escuelas y universidades, con efectos presumiblemente enormes sobre las condiciones psíquicas con las cuales debe hacer cuentas una sociedad. En esto Parsons ha visto una tercera revolución, junto a la política y la industrial. Pero todas estas descripciones tienen un error común. Captan correctamente un carácter pero luego lo extienden hasta constituirlo en caracterización primaria o únicamente determinante de la sociedad moderna.

¿Cómo se pueden corregir estas unilateralidades? Ciertamente no denegando su significado. Se trata indiscutiblemente de caracteres mediante los cuales la sociedad moderna se distingue de formaciones societarias más antiguas pero a una teoría de la sociedad es necesario pedirle que pueda explicar cómo y porqué en los más diversos ámbitos de la sociedad se llega a estas peculiaridades que caracterizan la modernidad. Esto no se puede hacer continuando (o negándose a hacerlo) los discursos filosóficos-literarios que a partir del iluminismo han acompañado y comentado el desarrollo de la sociedad moderna -en parte con entusiasmo enfático, en parte con repugnancia, luego otra vez repitiéndolos o resignándose. Más bien tenemos necesidad de una teoría más abstracta que mediante una solubilidad y capacidad recombinatoria que supere todas las experiencias, sea aplicable a la formación de la propiedad, dadas las condicio- 
nes capitalistas y a la política ordenada por estados regionales, a la educación organizada en las clases escolares y a la investigación científica.

Si a la base de esto se coloca una conceptualización teóricosistémica, se pueden concebir todas las características descritas como consecuencia de una autonomía de los sistemas societarios de función extremadamente elevada. Esto no debe significar que todo el actuar tiene lugar en un y solo en un sistema de función, pero la dinámica de la sociedad moderna es producida en estos sistemas. Instituciones más antiguas y multifuncionales, sobre todo las economías domésticas familiares como formas de vida normales y como forma de inclusión de los individuos en la sociedad son disueltas o reducidas a funciones propias. Así también una moral referida a esto pierde la propia plausibilidad. Y si la propiedad ha sido el concepto jurídico para la unidad de tales economías domésticas, así también la propiedad pierde su función central para la sociedad. Deviene inadecuado pensar que la sociedad consiste en propietarios que deben administrar ordenadamente sus economías domésticas, y correspondientemente también las instituciones de la sociedad a ellas ordenadas, como el comercio con mayor o menor excedente o las corporaciones como la iglesia, monasterios, universidades, mutualidades y gremios, se liberan de su anclaje en la sociedad. La sociedad compleja es ahora un sistema diferenciado funcionalmente y todo aquello que no se puede ordenar a este principio primario se puede explicar solo a partir de esto.

Una teoría de la sociedad de este tipo deberá sobre todo concentrarse en desarrollar la dinámica propia de los sistemas de función. La sociedad misma no puede ser concebida ni como si fuese una corporación capaz de regulación, ni como una masa caótica de acciones singulares. No se puede guiar políticamente, dado que la política misma es solo un sistema de función entre otros y, si se puede decir así, puede hacer solo política. Puede ser conocida científicamente, pero también esto significa que se la puede conocer solo científicamente, es decir, solo con la ayuda de construcciones (teorías) propias del sistema de la ciencia. Dado que cada planificación, cada regulación y cada conocimiento de la sociedad debe cumplirse en la sociedad y dado que operaciones de este tipo al mismo tiempo son eficaces y pueden ser observadas en la sociedad, la unidad del sistema se sustrae sea a la regulación, sea a la observación. La unidad del sistema es el operar de la reproducción autopoiética y su elaboración continua puede ser descrita solo como evolución. 
VI

Tal programa teórico no puede ser desarrollado aquí, ni siquiera puede ser presentado en modo adecuado. Debo entonces contentarme con bosquejar algunas consecuencias, estimulado por el tema "propiedad".

Si se considera la sociedad como sistema diferenciado, es necesario sobre todo tener distintos planos de observación. Ya no se pueden ordenar sin más instituciones sociales, como por ejemplo la propiedad, a la sociedad compleja, sino es necesario sobre todo entender qué sistema es primariamente pertinente. Para la sociedad moderna esto significa preguntarse qué sistema de función determina las formas que asume la propiedad, qué valor tiene en cada ocasión y sobre todo a partir de qué es influenciada y cambiada.

No se puede discutir el hecho de que aquí se deba referir a la economía. Naturalmente la propiedad es y permanece también como un concepto jurídico. Todo cambio de propiedad es al mismo tiempo transacción económica y jurídica. Para el derecho, sin embargo, la propiedad es un sector regulativo entre muchos otros, mientras para la economía es el código central. Esto es: el derecho decide con base en el código legal/nolegal sobre innumerables cuestiones, entre las cuales están aquellas concernientes a la propiedad. La economía no podría en cambio ni siquiera diferenciarse, no podría devenir un sistema de función de la sociedad, si no existiese la propiedad. Porque para toda transacción de la economía es presupuesto que se pueda establecer quién disponga de qué cosa. Dicho de modo diverso: en el sistema del derecho, la propiedad es componente de determinados programas que permiten establecer quién, en caso de disputa, tiene razón y quien está equivocado - quién está en lo legal y quién está en lo ilegal. En el sistema de la economía la propiedad es componente del código binario, el único que puede diferenciar el sistema. Aquí se trata de la división de la facultad dispositiva, de tener o no tener. Si no se pudiese distinguir entre tener y no tener no existiría economía alguna. Así como no existiría derecho alguno si no se pudiese distinguir entre legal e ilegal. Todos los programas de la economía, programas de producción, de inversión, de presupuesto (budget) de propietarios privados o estatales presuponen el código del sistema. Pueden ser hechos posibles solo mediante formación de propiedad. Esto vale sea en las economías privadas capitalistas, sea en las estatales capitalistas. Al interior de este esquema, pero solo en su interior, ciertamente luego pueden existir enormes diferencias, por ejemplo sobre la base de diversas medidas de la estatali- 
zación de los medios de producción, sobre la base de diversas conformaciones del sistema bancario o sobre la base de diversas formas de entrelazamiento y concentración privado-capitalistas. Pero estas son y permanecen diferencias secundarias, y precisamente en un doble sentido: (1) éstas no caracterizan diversos tipos de sociedad, sino por así decir diversos experimentos sociales al interior de una sociedad mundial compleja; y (2) estas no caracterizan siquiera diferentes sistemas de función de la economía, sino solo diversas formas de conexión entre código y programas al interior de un operante sistema de función mundial (de la sociedad mundial) de la economía.

A diferencia de otras representaciones de la semántica histórica de la propiedad, este esbozo basado sobre una teoría de la diferenciación es elaborado con dos distinciones, es decir (1) con la distinción entre diversos sistemas de función, sobre todo sistema de la economía y sistema del derecho, y (2) con la distinción entre codificación binaria y programación. Con la ayuda de estas herramientas de orientación, la propiedad es por así decir destronada semánticamente. Tal maniobra teórica puede naturalmente ser vista y liquidada como semántica política, sino como una "ideología" tardo-burguesa particularmente refinada. Todas las descripciones de la sociedad pueden ser observadas y descritas en la sociedad y la complejidad de la sociedad moderna consiste no por último en el hecho de que ella puede preparar más descripciones de sí misma que no pueden ser integradas. Los temas, los objetos, las cosas, han perdido su inamovible fijación del ser y ningún observador puede pretender la autoridad de una descripción única y correcta.

En esta situación, en las controversias se puede dejar si valorarla positivamente como pluralismo o si confiarse del todo al vértigo de la arbitrariedad postmoderna. No apenas existe un sistema diferenciado para la investigación científica en ello surgen sin embargo criterios específicos (referidos a programas propios = teorías) para la preferencia de descripciones. Uno de estos criterios es la consistencia y la complejidad cognitiva de una propuesta teórica. Si se parte de este criterio, la propuesta de concebir la propiedad como código del sistema de la economía y a partir de aquí estudiar la semántica tradicional de la propiedad así como las abstracciones jurídicas que de allí son derivadas y en fin las diferentes formas de la conexión entre codificación y programación, tiene algo que ofrecer. 
Permítanme brevemente resumir en forma de tesis:

1. El concepto de propiedad indica una hecho relativamente evidente. Esto explica su prominencia histórica en una sociedad de transición, que pasa a nuevas formas de diferenciación del sistema, que no las puede todavía reconocer y describir y que en cambio debía atenerse a transformaciones ya visibles y a esperanzas de progreso.

2. El derecho sigue la diferenciación creciente, la movilidad y la complejidad de la economía mediante abstracciones del concepto de propiedad, liberalizando la disponibilidad contractual y separando imperium (político) de dominium (económico). Se ve en varias ocasiones (y precisamente ya en la ética económica de la teología tardo-escolástica en los instrumentos regulativos mercantiles) que la economía no se puede regular jurídicamente, sino que debe regularse por sí misma en su propia dinámica. El derecho alista a tal fin solo conceptos globales, limitaciones de la disponibilidad y cláusulas de abuso, pero naturalmente no puede decidir que sería contrario a la ley si la economía no prospera.

3. Las consecuencias para una teoría sociológica (relativa a una teoría de la sociedad) de la economía no pueden ser ignoradas. Cierto es que la economía debe ser concebida a partir de su dinámica operativa y no a partir de la materia que ella elabora ni a partir del actuar humano individual. Mi impresión, además, es que las ciencias económicas mismas no están suficientemente preparadas para esto en la conceptualización del mercado ni en la conceptualización del dinero. La conceptualización aquí propuesta debería llevar a concebir la economía como un sistema de función autoreproducido y operativamente cerrado (autopoiético), que en dadas condiciones socioestructurales favorables surge y evoluciona si se puede distinguir entre tener-propiedad/no-tenerla y si este código regula las transacciones. Si es así, deben surgir cuestiones estructurales sobre la relación entre codificación y programación; y el problema será entonces aquél de responder a estas preguntas en un modo que sea compatible con la economía de mercado.

4. El objetivo de toda esta empresa teórica es sin embargo una descripción adecuada de la sociedad moderna y la cuestión, si y para quién una tal descripción sea aceptable, va mucho más allá de aquello que se puede aducir como argumentos estrictamente científicos. RM 
SOBRE EL AUTOR

Niklas Luhmann (1927-1998) es considerado uno de los sociólogos más importantes del último siglo. Su mayor aporte radica en la profundización de la teoría de sistemas en el ámbito de la sociología y el desarrollo de una teoría de la sociedad de pretensiones universalistas. Desarrolló gran parte de su carrera en la universidad de Bielefeld, Alemania, desde 1968 hasta su retiro en 1993, siendo el primer profesor que tuvo dicha universidad. Falleció el 6 de Noviembre de 1998. Niklas Luhmann escribió durante su vida más de cincuenta libros y cientos de artículos, por lo que es muy difícil una visión sucinta de su obra. Sin embargo podemos destacar entre sus últimos libros traducidos al español: La sociedad de la sociedad (Herder- Universidad Iberoamericana, 2007), Organización y decisión (Herder-Universidad Iberoamericana, 2010), El amor (Prometeo Libros, 2012) y Sociología política (Trotta, 2014).

\section{CONTACTO}

(Para consultas sobre su vida y obra)

Universität Bielefeld

Fakultät für Soziologie

Postfach 100131

33501, Bielefeld

Deutschland

Recibido: julio 2015

Aceptado: agosto 2015 